\title{
Reolysin is a novel reovirus-based agent that induces endoplasmic reticular stress-mediated apoptosis in pancreatic cancer
}

\author{
JS Carew ${ }^{1}$, CM Espitia ${ }^{1}$, W Zhao ${ }^{1}$, KR Kelly ${ }^{1}$, M Coffey ${ }^{2}$, JW Freeman ${ }^{1}$ and ST Nawrocki ${ }^{\star, 1}$
}

Activating mutation of KRas is a genetic alteration that occurs in the majority of pancreatic tumors and is therefore an ideal therapeutic target. The ability of reoviruses to preferentially replicate and induce cell death in transformed cells that express activated Ras prompted the development of a reovirus-based formulation for cancer therapy called Reolysin. We hypothesized that Reolysin exposure would trigger heavy production of viral products leading to endoplasmic reticular (ER) stress-mediated apoptosis. Here, we report that Reolysin treatment stimulated selective reovirus replication and decreased cell viability in KRas-transformed immortalized human pancreatic duct epithelial cells and pancreatic cancer cell lines. These effects were associated with increased expression of ER stress-related genes, ER swelling, cleavage of caspase-4, and splicing of XBP-1. Treatment with ER stress stimuli including tunicamycin, brefeldin A, and bortezomib (BZ) augmented the anticancer activity of Reolysin. Cotreatment with $\mathrm{BZ}$ and Reolysin induced the simultaneous accumulation of ubiquitinated and viral proteins, resulting in enhanced levels of ER stress and apoptosis in both in vitro and in vivo models of pancreatic cancer. Our collective results demonstrate that the abnormal protein accumulation induced by the combination of Reolysin and BZ promotes heightened ER stress and apoptosis in pancreatic cancer cells and provides the rationale for a phase I clinical trial further investigating the safety and efficacy of this novel strategy.

Cell Death and Disease (2013) 4, e728; doi:10.1038/cddis.2013.259; published online 18 July 2013

Subject Category: Cancer

Current treatments for pancreatic cancer have little effect on the aggressive course of this disease. The 5-year survival rate remains at only $5 \%$, and new approaches are urgently needed to improve the survivorship of patients with pancreatic cancer. A better understanding of the biology of pancreatic cancer will enable the design of more effective therapeutic strategies to improve clinical outcomes for patients. Constitutive Ras signaling is a prevalent phenomenon that occurs in diverse tumor types and is associated with transformation, proliferation, and reduced sensitivity to conventional chemotherapy. ${ }^{1}$ Activating mutations in the Ras gene are present in $90 \%$ of all cases of pancreatic cancer and have been linked to many aspects of the pathogenesis of this disease. ${ }^{2,3}$ Given this, mutant Ras is a very attractive target for selective pancreatic cancer therapy.

Reoviruses are naturally occurring viruses that are nonpathogenic and have been reported to specifically replicate in cancer cells with an activated Ras pathway but not in normal tissue. $^{4-6}$ To take advantage of this observation therapeutically, the reovirus-based anticancer agent Reolysin was recently developed and has already progressed into clinical trials, ${ }^{7-11}$ but its mechanism(s) of action remains unclear. The preferential replication of reovirus in transformed cells with activated Ras is due to the inhibition of double-stranded RNA-activated protein kinase (PKR) activity. ${ }^{12,13}$ In untransformed (normal) cells, PKR is autophosphorylated and activated by viral products, which leads to phosphorylation of the eukaryotic initiation factor $2 \alpha$-subunit (eif $2 \alpha$ ) and inhibition of viral protein synthesis. Phosphorylation of eif $2 \alpha$ activates a signaling pathway termed the integrated stress response where upregulation of activating transcription factor 4 (ATF4) is a key mediator. Activation of Ras inhibits PKR and subsequent eif2 $\alpha$ phosphorylation and therefore allows translation to continue, resulting in an accumulation of viral particles inside cancer cells. We hypothesized that unchecked viral replication in Ras-activated pancreatic cancer cells would promote endoplasmic reticular (ER) stress and apoptosis.

In our previous studies, we demonstrated that treatment with the proteasome inhibitor bortezomib (BZ) generated a

\footnotetext{
${ }^{1}$ Department of Medicine, Cancer Therapy and Research Center, Institute for Drug Development, University of Texas Health Science Center at San Antonio, San Antonio, TX, USA and ${ }^{2}$ Oncolytics Biotech Inc., Calgary, Alberta, Canada

${ }^{*}$ Corresponding author: ST Nawrocki, Department of Medicine, Cancer Therapy and Research Center, Institute for Drug Development, University of Texas Health Science Center at San Antonio, 7979 Wurzbach Road, San Antonio, TX 78229, USA. Tel: + 210450 3894; Fax: + 210450 3861; E-mail: Nawrocki@uthscsa.edu Keywords: Reolysin; endoplasmic reticular stress; bortezomib; reovirus; pancreatic cancer

Abbreviations: BZ, bortezomib; eif2 $\alpha$, eukaryotic initiation factor $2 \alpha$-subunit; ER stress, endoplasmic reticular stress; FBS, fetal bovine serum; HPNE, human pancreatic nestin expressing; IHC, immunohistochemistry; i.v., intravenous; MTT, 3-(4,5-dimethylthiazol-2-yl)-2,5-diphenyltetrazolium bromide; PERK, PKR-like endoplasmic reticulum kinase; PFU, plaque-forming units; PI-FACS, propidium iodide fluorescence-activated cell sorting; PKR, double-stranded RNA-activated protein kinase; qRT-PCR, quantitative real-time polymerase chain reaction; S.D., standard deviation; TUNEL, terminal deoxyribonucleotide transferase-mediated dUTP nick end labeling; UPR, unfolded protein response

Received 18.2.13; revised 07.6.13; accepted 17.6.13; Edited by C Munoz-Pinedo
} 
substantial accrual of ubiquitin-conjugated proteins and induced ER stress-mediated apoptosis in both in vitro and in vivo models of pancreatic cancer. ${ }^{14-16}$ Considering that abnormal protein buildup can trigger pancreatic cancer cell death, the simultaneous accumulation of ubiquitinated proteins and viral products may be especially toxic to pancreatic cancer cells. Furthermore, the high protein synthesis rates of pancreatic cancer cells with activated Ras compared with the low protein synthesis rates of normal cells suggest that this therapeutic strategy may selectively kill pancreatic cancer cells via ER stress-mediated cell death. In this study, we demonstrate that Reolysin induces an accumulation of viral products in pancreatic cancer cells with activated Ras, which results in ER stress and apoptosis. Further stimulation of ER stress with conventional ER stress-inducing agents (i.e., tunicamycin) or BZ augments the anticancer activity of Reolysin in both in vitro and in vivo models of pancreatic cancer.

\section{Results}

Reovirus selectively replicates in KRas-transfected immortalized pancreatic epithelial cells. Reovirus has been reported to selectively replicate in cancer cells with an activated Ras pathway. ${ }^{12,17,18}$ To investigate potential Ras-dependent selective replicative capacity in pancreatic cells, we quantified the levels of reovirus in control (KRas negative) and KRas-transfected immortalized normal pancreatic epithelial (human pancreatic nestin expressing (HPNE)) cells following treatment with Reolysin (Figure 1a). As expected based on earlier studies conducted in other cell types, exposure to Reolysin resulted in preferential reovirus replication in KRas-positive HPNE cells (Figure $1 \mathrm{~b}$ ). Consistent with the high abundance of viral proteins in the KRas-transfected cells, Reolysin treatment induced the expression of ER stress-related genes, including GRP78/ $B i P, G A D D 34$, and $C H O P$, and also increased the levels of
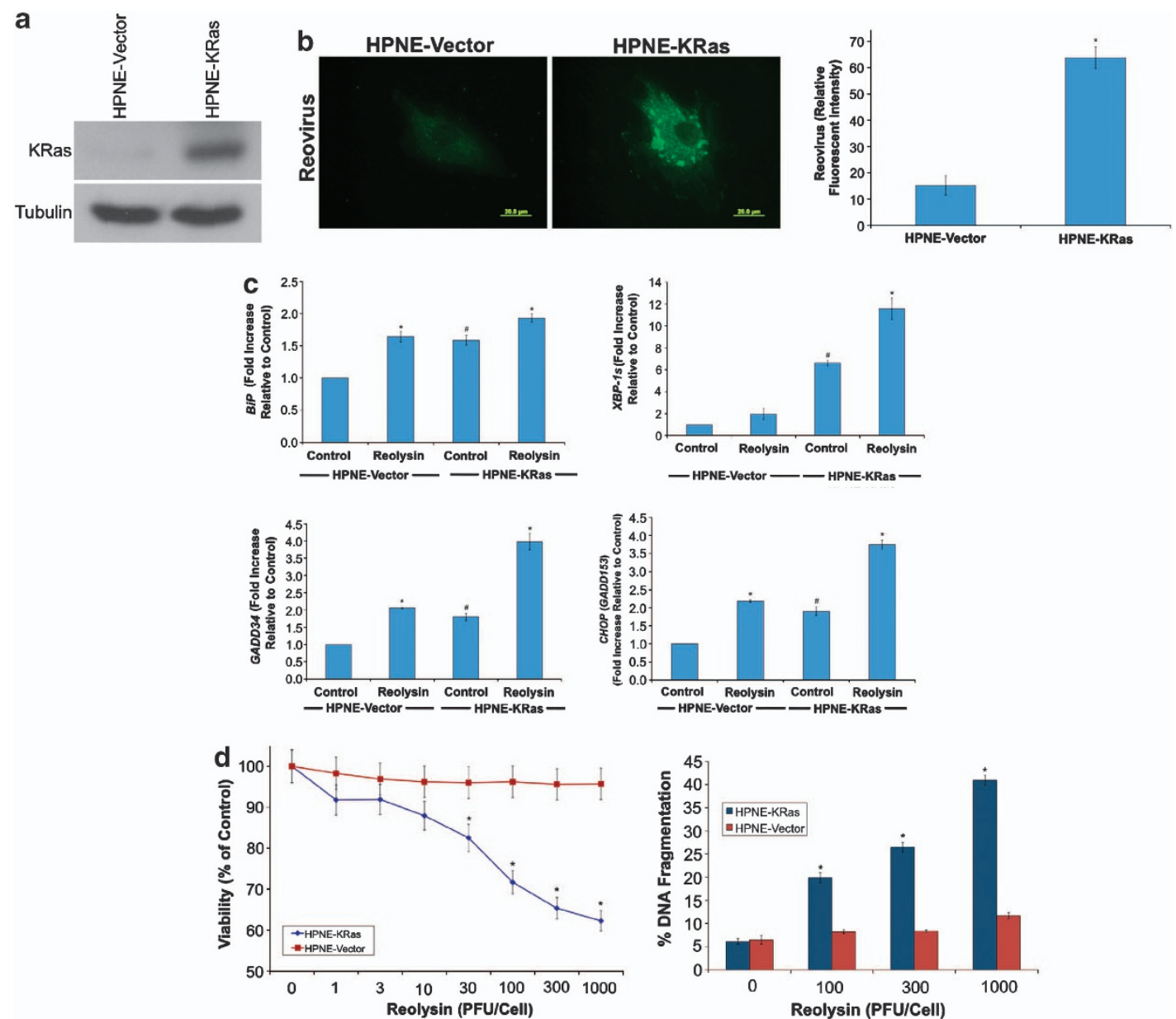

Figure 1 Reovirus preferentially replicates in KRas-transfected immortalized normal human pancreatic epithelial cells. (a) KRas transfected HPNE cells. Immunoblotting demonstrates KRas levels in HPNE cells. (b) Reovirus replicates preferentially in HPNE-KRas cells. Cells were treated with Reolysin for $48 \mathrm{~h}$ and stained with an anti-reovirus antibody. Immunocytochemistry reveals reovirus replication in KRas-transfected cells. Fluorescent intensity was quantified in HPNE and HPNE-KRas cells using Image-Pro Plus software version 6.2.1. Mean \pm S.D., $n=5$. *Indicates a significant difference compared with HPNE-vector cells. (c) KRas-transfected cells display higher levels of ER stress-related gene expression that can be further induced with reovirus exposure. HPNE-vector and HPNE-KRas cells were treated with 100 plaque-forming units (PFU)/cell Reolysin for $48 \mathrm{~h}$. Gene expression was determined by qRT-PCR. Mean \pm S.D., $n=3$. "Represents a significant difference compared with vector control cells. *Indicates a significant difference compared with corresponding controls. (d) HPNE-KRas cells are sensitive to Reolysin-mediated cell death. Cells were treated for $72 \mathrm{~h}$ with the indicated concentrations of Reolysin, and cell viability was determined by MTT assay (left panel). Cells were treated for $48 \mathrm{~h}$ with Reolysin, and apoptosis was measured by PI-FACS analysis (right panel). Mean \pm S.D., $n=3$. ${ }^{*}$ Indicates a significant difference compared with HPNE-vector cells treated with the same concentration of Reolysin $P<0.05$ 
the spliced form of $X B P-1$, suggesting induction of ER stress (Figure 1c). Notably, the basal levels of all of these genes were significantly higher in the KRas-expressing cells, indicating that cells with activated Ras may be under constitutive ER stress (Figure 1c). Measurement of other chaperones, including calreticulin, PDI, and ERp57, revealed that the levels were also significantly higher in KRastransfected cells (Supplementary Figure 1). However, only ERp57 was significantly induced following Reolysin treatment. Interestingly, BiP, GADD34, CHOP, and ERp57 levels were also increased in HPNE vector cells treated with Reolysin, albeit to a much lesser degree than in KRasexpressing cells. These data suggest that reovirus infection may also induce some degree of ER stress in wild-type Ras cells. This is not surprising as these non-transformed cells are not completely impervious to reovirus infection. Given that KRas-transfected cells have higher basal levels of ER stress than wild-type cells, further induction of ER stress with Reolysin may trigger a threshold point leading to apoptosis. In agreement with the elevated reovirus replication and ER stress induction we observed in KRas-transfected cells, Reolysin treatment selectively reduced cell viability and induced apoptosis in the HPNE-KRas cells compared with that in HPNE controls (Figure 1d).

Reolysin induces ER stress and apoptosis in pancreatic cancer cells. Given that reovirus has been reported to preferentially replicate in cells with an activated Ras pathway and that Ras is mutated in the majority of pancreatic cancers, we hypothesized that Reolysin may have significant activity against this tumor type. We first evaluated the ability of reovirus to replicate in the KRas-mutant Panc- 1 pancreatic cancer cell line. Immunocytochemistry and electron microscopy revealed a large intracellular accumulation of reovirus following $48 \mathrm{~h}$ treatment with Reolysin (Figure 2a). Prior studies show that reovirus does not activate PKR in Ras-mutated cells. We investigated whether this was also true for PKR-like endoplasmic reticulum kinase (PERK). Immunoblotting demonstrated that Reolysin treatment does not result in PERK or eif $2 \alpha$ phosphorylation (Figure $2 b$ ), which is consistent with reovirus exposure not suppressing translation in pancreatic cancer cells. However, transmission electron microscopy demonstrated significant ER swelling, indicating that ER stress may be induced in reovirus-infected cells (Figure 2c). In agreement with this observation, reovirus infection led to a dose-dependent increase in intracellular calcium levels (Figure 2d). Moreover, reovirus exposure significantly increased the expression of ER stress-related genes including GRP78/BiP, XBP-1s, GADD34, and CHOP/ GADD153 in the Panc-1 pancreatic cancer cell line in a manner that was consistent with our gene expression data obtained in Reolysin-treated HPNE-KRas cells (Figure 2e). However, no significant induction in other chaperone proteins (calreticulin, PDI, and ERp57) was observed (Supplementary Figure 2), indicating that reovirus infection may selectively induce BiP chaperone protein expression. Similar results were demonstrated by immunoblotting (Figure 2f), which showed that $\mathrm{CHOP}, \mathrm{GADD} 34$, and $\mathrm{BiP}$ were significantly induced following Reolysin treatment. Collectively, these data demonstrate that Reolysin treatment induces many of the hallmark features of ER stress in pancreatic cancer cells.

We next evaluated the anticancer activity of Reolysin in a panel of human pancreatic cancer cell lines. 3-(4,5-Dimethylthiazol-2-yl)-2,5-diphenyltetrazolium bromide (MTT) assay demonstrated a dose-dependent decrease in cell viability following Reolysin treatment in four KRas-mutant cell lines (Figure 3a). To investigate whether apoptotic cell death contributed to the decreased viability we observed following Reolysin exposure, we measured caspase-3 cleavage and DNA fragmentation by immunoblotting and propidium iodide fluorescence-activated cell sorting (PI-FACS) analysis (Figures $3 \mathrm{~b}$ and $\mathrm{c}$ ). Both of these experiments revealed that Reolysin treatment triggered significant levels of apoptosis. Earlier investigations have demonstrated that apoptosis following ER stress in humans may be initiated via activation of the ER-resident caspase-4. ${ }^{14,19}$ Here, we show that caspase-4 is cleaved to its active form after Reolysin treatment, suggesting that ER stress-mediated apoptosis may significantly promote cell death following reovirus infection (Figure $3 b$ ).

ER stress inducers augment the anticancer activity of Reolysin. Reolysin is currently in clinical trials for the treatment of many cancer types, including pancreatic cancer. We hypothesized that further stimulation of ER stress may augment the anticancer activity of Reolysin. To test this hypothesis, we treated Panc-1 cells with two well-established ER stress inducers, brefeldin $A$ and tunicamycin, in the presence or absence of Reolysin (Figure 3d). Both of these agents significantly enhanced the pro-apoptotic activity of Reolysin, demonstrating that agents that stimulate ER stress may be useful for combination therapy with Reolysin.

Proteasome inhibitors have been previously reported to induce ER stress via the accumulation of undegraded ubiquitinated protein aggregates. ${ }^{14,16,20-22}$ We hypothesized that the simultaneous accumulation of ubiquitinated aggregates and viral products in cells treated with Reolysin and the proteasome inhibitor BZ would result in heightened levels of ER stress and apoptosis. Reovirus and ubiquitinated protein accumulation was visualized by confocal and electron microscopy. The combination of Reolysin and BZ led to a dual accumulation of reovirus and ubiquitinated proteins in pancreatic cancer cells that was markedly greater than the protein buildup that was achieved by either monotherapy (Figures $4 \mathrm{a}$ and $\mathrm{b}$ ). Consistent with the high levels of viral and ubiquitinated proteins present inside these cells, simultaneous treatment with Reolysin and BZ significantly reduced pancreatic cancer cell viability and augmented apoptosis (Figures $4 c$ and $d$ ).

Reolysin and BZ cooperate to stimulate increased ER stress-mediated apoptosis. To further characterize the pharmacodynamic effects of Reolysin and BZ on pancreatic cancer cells, we measured markers of ER stress following single-agent and combination treatments. As the ER is the major intracellular calcium store, agents that stimulate ER stress frequently promote an increase in cytosolic calcium levels that occurs due to the inability of stressed ER to retain this important ion. Consistent with this 

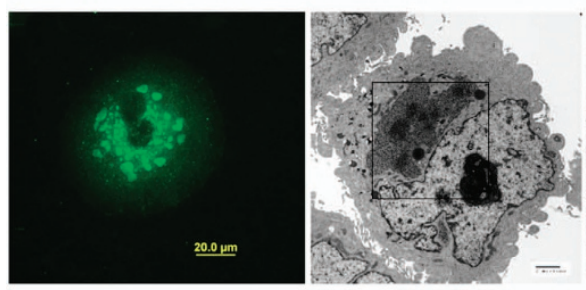

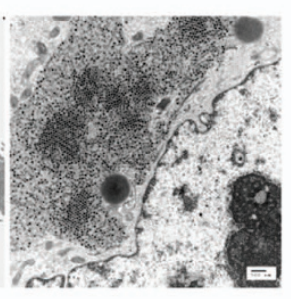

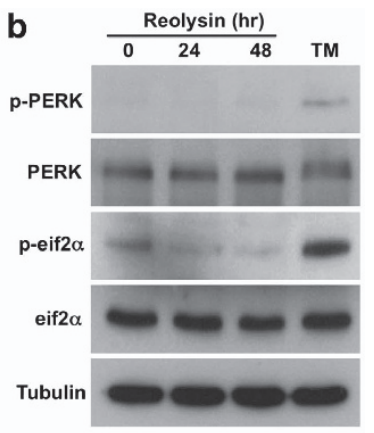

c
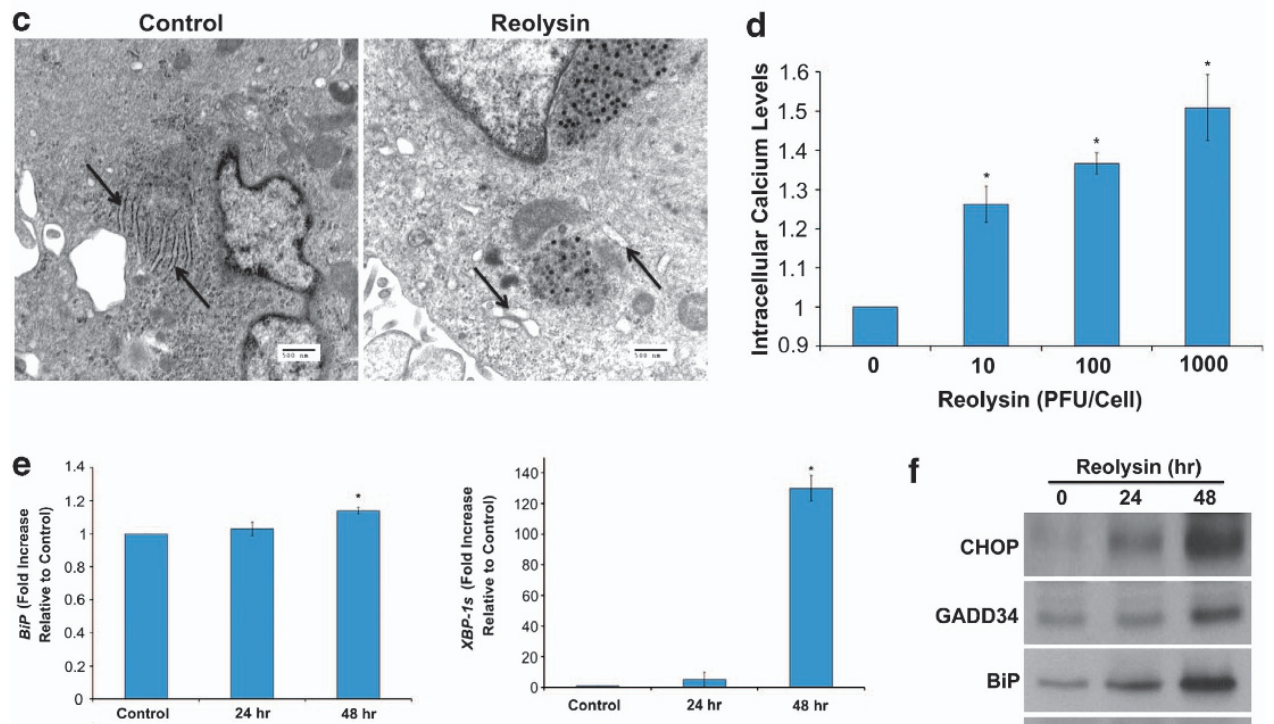

f

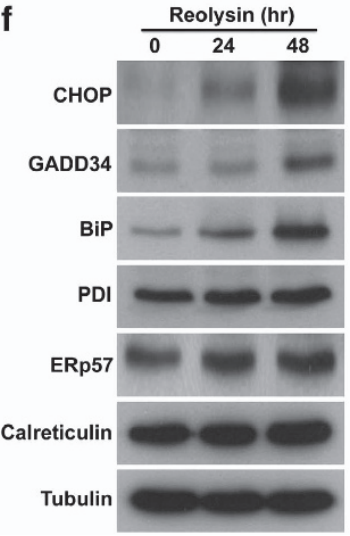

Figure 2 Reolysin induces ER stress. (a) Reovirus replication in Panc-1 cells. Cells were treated with $100 \mathrm{PFU} / \mathrm{cell}$ Reolysin for $48 \mathrm{~h}$. Reovirus replication was detected by immunocytochemistry and electron microscopy. (b) Reolysin does not promote PERK or eif2 $\alpha$ phosphorylation. Panc-1 cells were treated with 100 PFU/cell Reolysin for 24 and $48 \mathrm{~h}$ or with $5 \mu \mathrm{g} / \mathrm{ml}$ tunicamycin $(24 \mathrm{~h}$ ) as a positive control. Proteins were detected by immunoblotting. (c) Reolysin promotes ER swelling. Panc-1 cells were treated with $100 \mathrm{PFU} / \mathrm{cell}$ Reolysin for $48 \mathrm{~h}$, and ER morphology was visualized by electron microscopy. Arrows denote endoplasmic reticulum. (d) Reolysin treatment increases intracellular calcium levels. Panc-1 cells were treated with the indicated amounts of Reolysin for $16 \mathrm{~h}$, and intracellular calcium levels were detected by calcium green-1 staining and flow cytometry. Mean \pm S.D., $n=3$. ${ }^{*}$ Represents a significant difference compared with controls. (e) qRT-PCR analysis of BiP, GADD34, CHOP, and XBP-1s expression in Panc-1 cells. Cells were treated with $100 \mathrm{PFU} / \mathrm{cell}$ Reolysin for 24 and $48 \mathrm{~h}$ and then harvested for analysis. Levels of mRNAs were standardized to the expression of GAPDH. Mean \pm S.D., $n=3$. *Indicates a significant difference from the control. $P<0.05$. (f) Immunoblotting analysis of CHOP, GADD34, BiP, PDI, ERp57, and calreticulin. Panc-1 cells were treated with $100 \mathrm{PFU} / c e l l$ Reolysin for 24 or $48 \mathrm{~h}$. ER-related protein expression was measured by immunoblotting

phenomenon, both Reolysin and BZ single-agent treatment increased cytosolic calcium levels. This effect was further enhanced by the combination of both agents (Figure 5a). In addition, quantitative real-time PCR (qRT-PCR) demonstrated that the levels of GRP78/BiP, XBP-1s, GADD34, and $C H O P$ were all significantly induced by each single agent and further increased by combination therapy (Figure 5b). As expected, caspase-4 cleavage was also increased following Reolysin and BZ treatment and directly correlated with enhanced cleavage of caspase-3 (Figure 5c). To establish the mechanistic role of caspase-4 in Reolysin- and BZ-induced apoptosis, siRNA was used to knockdown its expression (Figure 5d). Cells with reduced caspase-4 levels were significantly less sensitive to apoptosis induced by Reolysin, BZ, or the combination (Figure $5 \mathrm{~d}$ ). To further show that Reolysin and BZ stimulate ER stress, we measured the levels of caspase-12, an ER-resident caspase that is required for ER stress-mediated apoptosis in murine cells. ${ }^{23}$ Consistent with our earlier data generated in human pancreatic cancer models, treatment with the Reolysin and BZ combination resulted in a strong increase in caspase- 12 cleavage in murine L929 fibrosarcoma cells. In addition, the 
combination induced significantly greater levels of apoptosis in these cells compared with either single-agent treatment (Supplementary Figure 3).

BZ augments the activity of Reolysin in vivo. We next conducted a xenograft study to investigate the potential

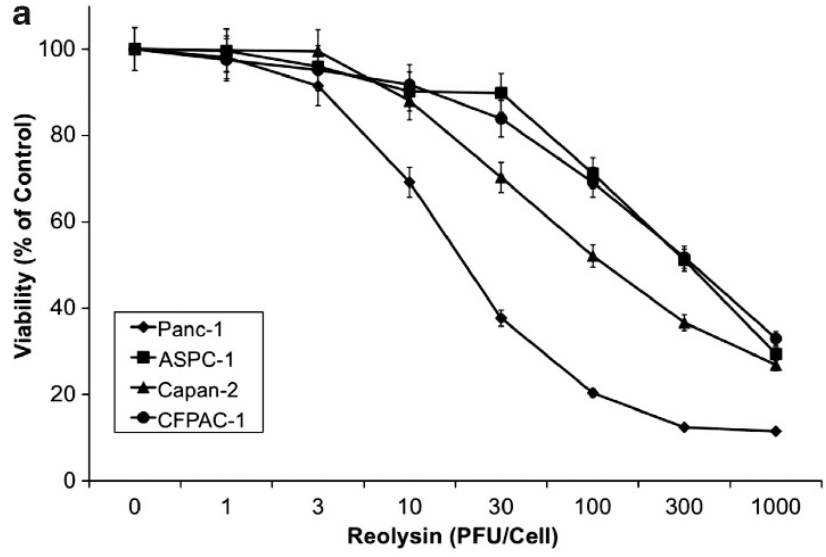

b

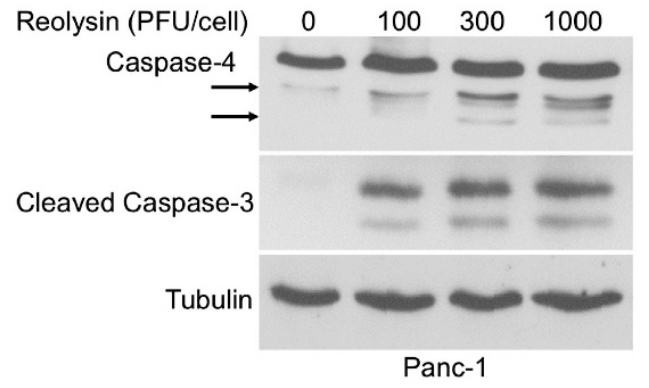

C

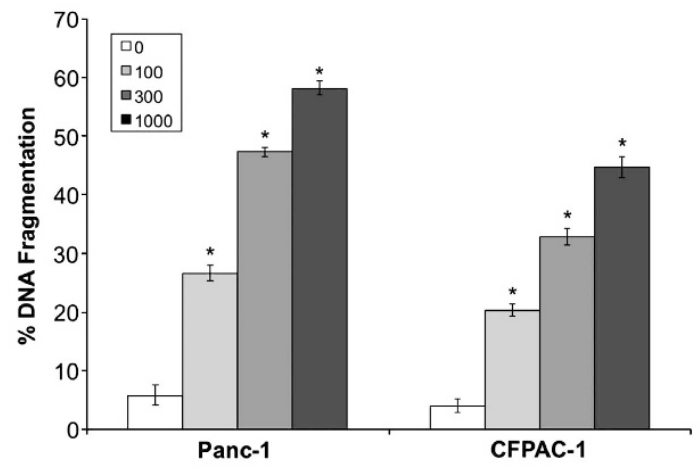

d

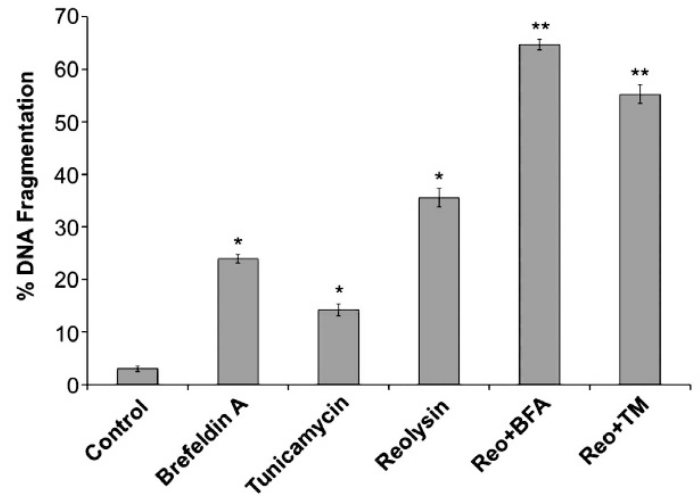

therapeutic benefit of the Reolysin and BZ combination. A mouse model of pancreatic cancer was generated by implanting Panc-1 cells into nude mice. Tumor-bearing animals were randomized into treatment groups and administered vehicle (PBS), $0.5 \mathrm{mg} \mathrm{BZ}$ per $\mathrm{kg}$ intravenous (i.v.) Q3D, $5 \times 10^{8}$ TCID $_{50}$ Reolysin i.v. Q7D, or both agents for 5 weeks. Treatment with either single agent significantly antagonized tumor progression (Figure 6a, left). However, the Reolysin and BZ combination led to a dramatic decrease in tumor burden (Figure 6a, left) that was markedly greater than what was achieved with either monotherapy. Moreover, the combination treatment was well tolerated as no significant animal weight loss was observed at the completion of the study (day 38) (Figure 6a, right). We next evaluated whether we could detect the presence of reovirus inside Panc-1 tumors treated with the combination. Consistent with our in vitro data, reovirus was readily detected in Reolysin-treated tumors by electron microscopy (Figure 6b). Notably, reovirus was present to an equivalent extent in tumors treated with Reolysin alone and Reolysin plus BZ, suggesting that BZ does not alter reovirus replication. In agreement with our related in vitro assays, immunohistochemical analysis of tumor sections revealed a significant increase in $\mathrm{BiP}$ expression (Figure 6c) and apoptosis (Figure 6d) in mice treated with Reolysin and BZ. Collectively, these data provide evidence that Reolysin induces ER stress and possesses significant activity in models of Ras-activated pancreatic cancer that can be augmented via further induction of ER stress with $B Z$.

\section{Discussion}

Reovirus is an oncolytic virus that has been reported to selectively replicate in cells with an activated Ras pathway. ${ }^{12,13}$ Given the very high prevalence of Ras mutations in pancreatic cancer, patients with KRas-positive pancreatic tumors may be intrinsically sensitive to reovirus treatment. The results of our current investigation are consistent with previous findings in different cancer types that also demonstrated that reovirus preferentially replicates in Ras-transformed cells. ${ }^{12,17,24,25}$ Our data show that reovirus selectively replicated in KRas-transfected normal pancreatic epithelial cells versus KRas-negative HPNE cells, indicating that Ras activity is a key determinant regulating Reolysin sensitivity. Although it is clear that Reolysin possesses significant activity

Figure 3 Reolysin promotes caspase-4 processing and apoptosis and sensitizes cells to ER stress-mediated apoptosis. (a) Reolysin decreases cell viability in a panel of pancreatic cancer cell lines. Cells were treated with the indicated concentrations of Reolysin for $72 \mathrm{~h}$. Cell viability was measured by MTT assay. Mean \pm S.D., $n=3$. (b) Reolysin promotes cleavage of caspase-4 and caspase-3. Panc-1 cells were treated with the indicated concentrations of Reolysin for $48 \mathrm{~h}$, and caspase cleavage was measured by immunoblotting. Arrows denote cleaved caspase-4 fragments. (c) Reolysin induces apoptosis. Panc-1 and CFPAC1 cells were treated with Reolysin for $48 \mathrm{~h}$. Apoptosis was measured by PI-FACS analysis. Mean \pm S.D., $n=3$. ${ }^{*}$ Indicates a significant difference compared with controls. (d) Reolysin augments ER stress-mediated apoptosis. Panc-1 cells were treated for $48 \mathrm{~h}$ with $300 \mathrm{PFU} / \mathrm{cell}$ Reolysin, $5 \mu \mathrm{g} / \mathrm{ml}$ tunicamycin, $5 \mu \mathrm{M}$ brefeldin A, and combinations. Mean \pm S.D., $n=3$. ${ }^{*}$ Indicates a significant difference compared with controls; **indicates a significant difference compared with either single-agent treatment $P<0.05$ 
a
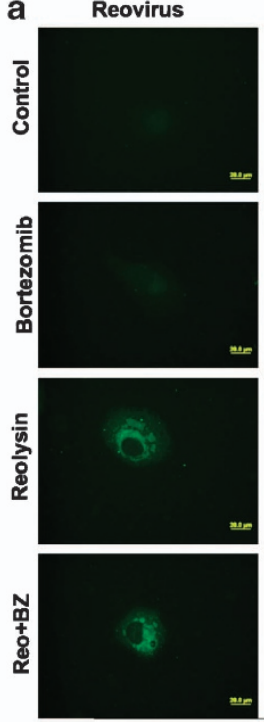

Ubiquitin
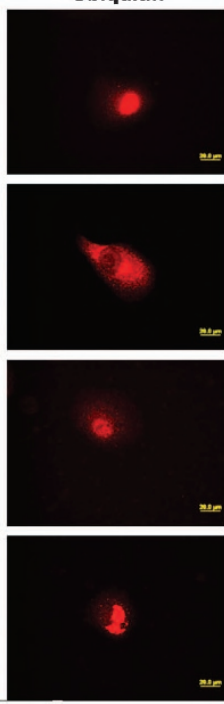

Overlay
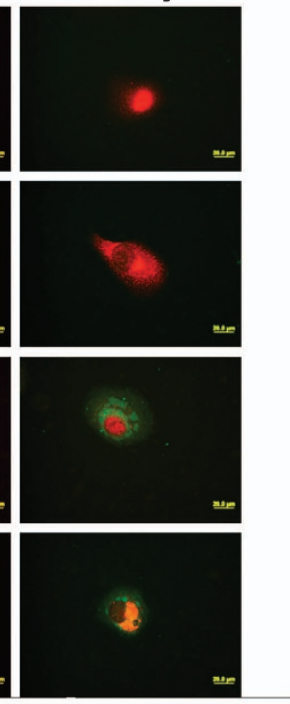
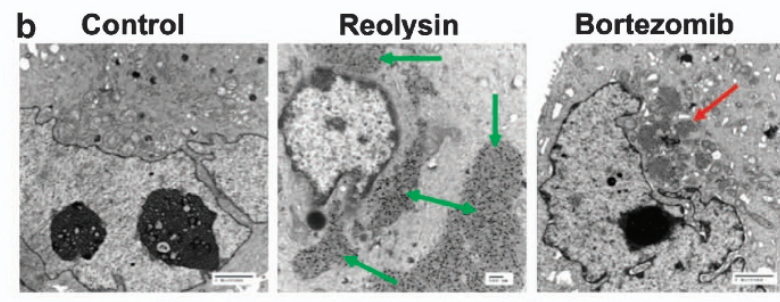

Reo+BZ
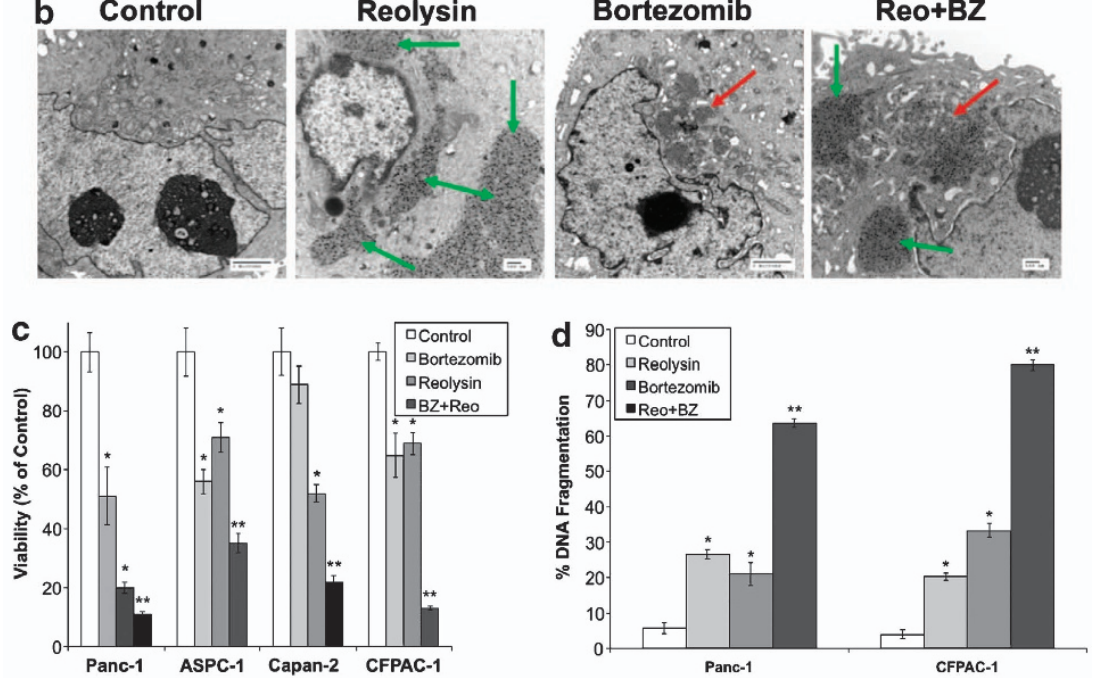

Figure 4 Reolysin augments the anticancer activity of BZ. (a and $\mathbf{b})$ Reolysin and BZ stimulate reoviral and ubiquitinated protein accumulation. Cells were treated with $100 \mathrm{PFU} / \mathrm{cell}$ Reolysin and $10 \mathrm{nM} \mathrm{BZ}$ for $48 \mathrm{~h}$. Protein accumulation was visualized by (a) immunocytochemistry and (b) electron microscopy. Red arrows indicate ubiquitinated protein aggregates, and green arrows depict reovirus replication. (c) Reolysin and BZ reduce cell viability. Pancreatic cancer cells were treated with $100 \mathrm{PFU} / \mathrm{cell}$ Reolysin and $10 \mathrm{nM} \mathrm{BZ}$ for $72 \mathrm{~h}$. Cell viability was measured by MTT assay. *Indicates a significant difference from controls and * ${ }^{*}$ denotes a significant difference compared to either single agent treatment group $(P<0.05)$. (d) Reolysin and BZ induce apoptosis. Cells were treated with $100 \mathrm{PFU} /$ cell Reolysin and $10 \mathrm{nM} \mathrm{BZ}$ for $48 \mathrm{~h}$. Apoptosis was determined by PI-FACS analysis. Mean \pm S.D., $n=3$. *Represents a significant difference from controls. ${ }^{* *}$ Indicates a significant difference compared with single-agent treatment groups $P<0.05$

against Ras-activated cancer cells, the exact mechanisms of Reolysin-mediated cell death need further investigation. In this study, we show that reovirus accumulation stimulates many of the hallmark features of ER stress, including ER swelling, increased cytosolic calcium levels, elevated expression of ER stress-related genes, and processing of the ER-resident capase-4. Previous studies have shown that processing of caspase- 4 is a signature characteristic of ER stress-mediated apoptosis in human cells. ${ }^{14,19,26}$ Further analysis revealed that knockdown of caspase-4 significantly reduced Reolysin-induced apoptosis, demonstrating that stimulation of ER stress is an important mediator of cell death following reovirus infection. In addition, our results suggest that cells with high Ras activity may be under constitutive ER stress as the introduction of KRas to pancreatic epithelial cells increased the basal expression of several ER stress-related genes. Although reovirus infection also increased some ER stress-related gene expression levels in wild-type Ras cells, it was not associated with reduced cell viability or increased apoptosis. Therefore, Ras-activated cells may be under constitutive ER stress, and further stimulation of ER stress with Reolysin may push the cells beyond a threshold point, resulting in ER stress-mediated apoptosis. These results are consistent with prior reports demonstrating that oncogenic Ras activation disrupts cellular redox status and induces ER stress. $^{27,28}$ 

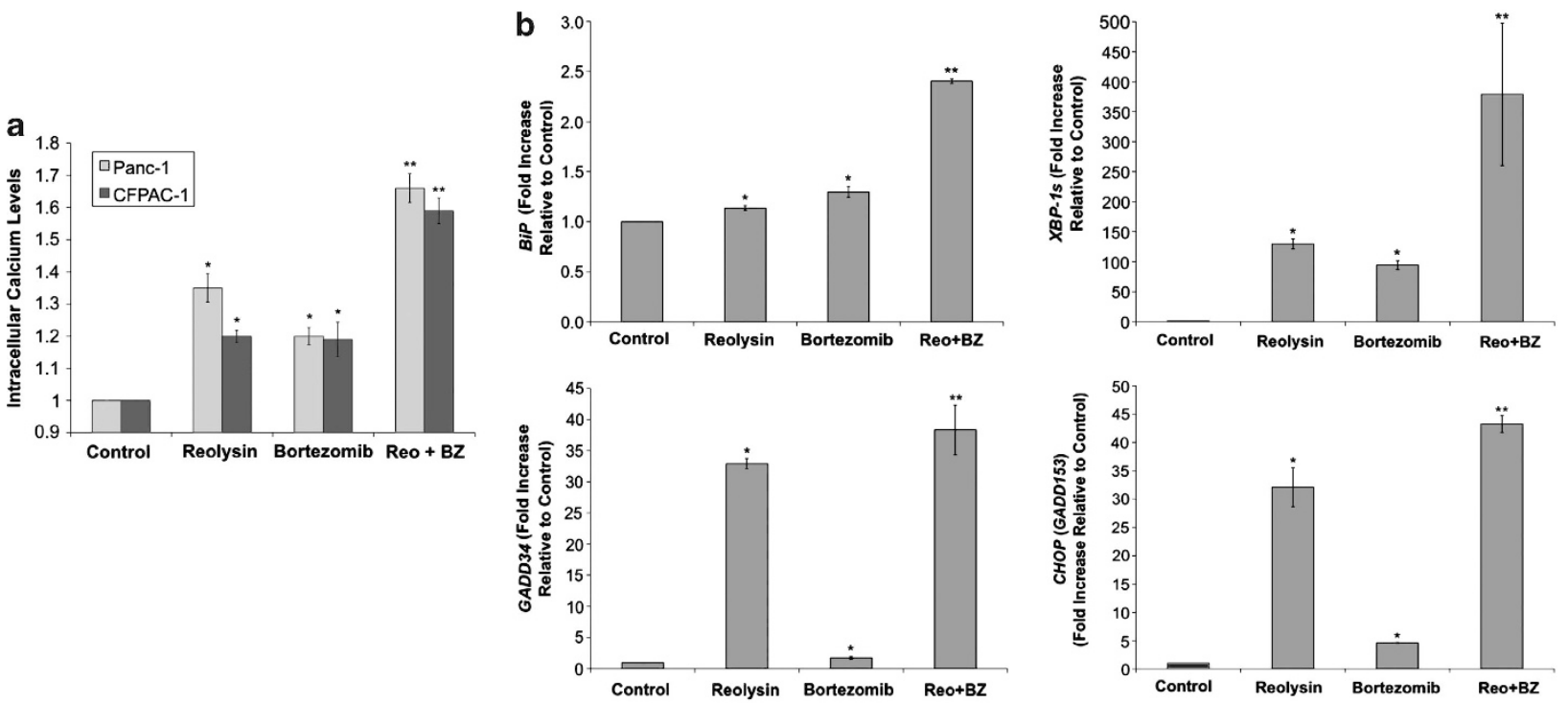

C
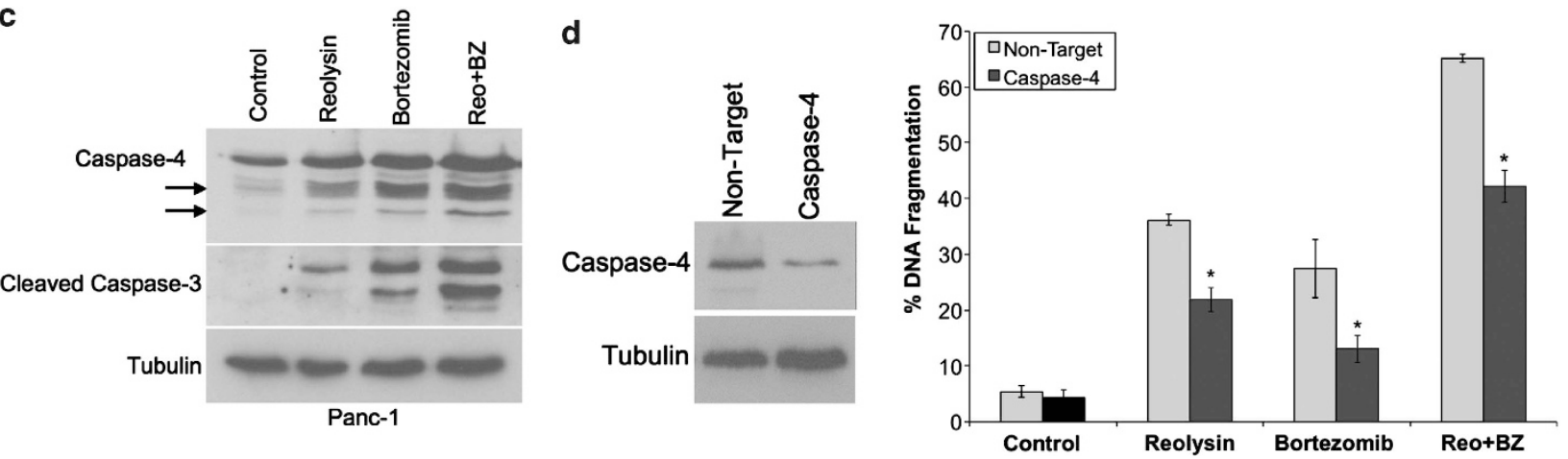

Figure 5 Reolysin enhances BZ-mediated ER stress and apoptosis. (a) The Reolysin and BZ combination increases intracellular calcium levels. Cells were treated with $100 \mathrm{PFU} /$ cell Reolysin and $10 \mathrm{nM} \mathrm{BZ}$ for $16 \mathrm{~h}$, and intracellular calcium levels were detected by calcium green-1 staining and flow cytometry. Mean \pm S.D., $n=3$. *Represents a significant difference compared with controls, and **indicates a significant difference compared with either single-agent treatment. (b) Reolysin augments BZ-induced increases in ER stress-related gene expression. Panc-1 cells were treated with $100 \mathrm{PFU} /$ cell Reolysin and $10 \mathrm{nM} \mathrm{BZ}$ for $48 \mathrm{~h}$ and then harvested for analysis. Levels of mRNA were standardized to the expression of GAPDH. Mean \pm S.D., $n=3$. ${ }^{*}$ Indicates a significant difference from the control. ${ }^{*}$ Indicates a significant difference compared with single-agent treatment groups. (c) Reolysin enhances BZ-mediated cleavage of caspase-4 and caspase-3. Cells were treated with $100 \mathrm{PFU} / \mathrm{cell}$ Reolysin and $10 \mathrm{nM}$ BZ for $48 \mathrm{~h}$. Caspase cleavage was measured by immunoblotting. Arrows denote caspase-4 cleavage fragments. (d) Knockdown of caspase-4 reduces Reolysin- and BZ-induced apoptosis. siRNA-mediated knockdown of caspase-4 was determined by immunoblotting at $72 \mathrm{~h}$ post transfection. Cells were treated with Reolysin and BZ $24 \mathrm{~h}$ post transfection for $48 \mathrm{~h}$. Apoptosis was measured by PI-FACS analysis. Mean \pm S.D., $n=3$. *Indicates a significant difference compared with non-target-transfected cells treated under the same conditions $P<0.05$

Although Reolysin has significant anticancer activity when administered as a monotherapy, several preclinical and clinical studies suggest that it may be best utilized in combination with standard chemotherapy. ${ }^{29-31}$ To select the optimal partner agents for combination chemotherapy, it is important to understand the mechanisms underlying the anticancer activity of Reolysin. Based on our initial data demonstrating that Reolysin stimulates ER stress, we hypothesized that classical ER stress inducers tunicamycin and brefeldin $A$ and the proteasome inhibitor BZ would augment Reolysin-mediated apoptosis through further ER stress induction. In agreement with this hypothesis, all three agents significantly enhanced Reolysin-mediated apoptosis. We focused our subsequent experiments on the combination of Reolysin and BZ as BZ is an FDA-approved drug for cancer therapy. Reolysin in combination with $B Z$ induced high levels of ER stress due to the dual accumulation of both ubiquitin-conjugated protein aggregates and viral protein products. Importantly, the high levels of proteotoxicity caused by this combination resulted in enhanced caspase-4/caspase12 processing and apoptosis.

To further evaluate the potential benefit of this therapeutic approach, we conducted a xenograft study using the Panc-1 tumor model. Consistent with our in vitro data, a dramatic reduction in tumor burden was noted in animals treated with the combination of Reolysin and BZ compared with that in animals treated with either single-agent therapy. In addition, the combination was very well tolerated, with no significant animal weight loss or other toxicity observed in the combination-treated mice. Analysis of tumor samples revealed an increase in ER stress and apoptosis in the combinationtreated tumors. This therapeutic approach is consistent with a prior study demonstrating that inhibition of the unfolded protein response (UPR) mediator IRE1 improved the efficacy 

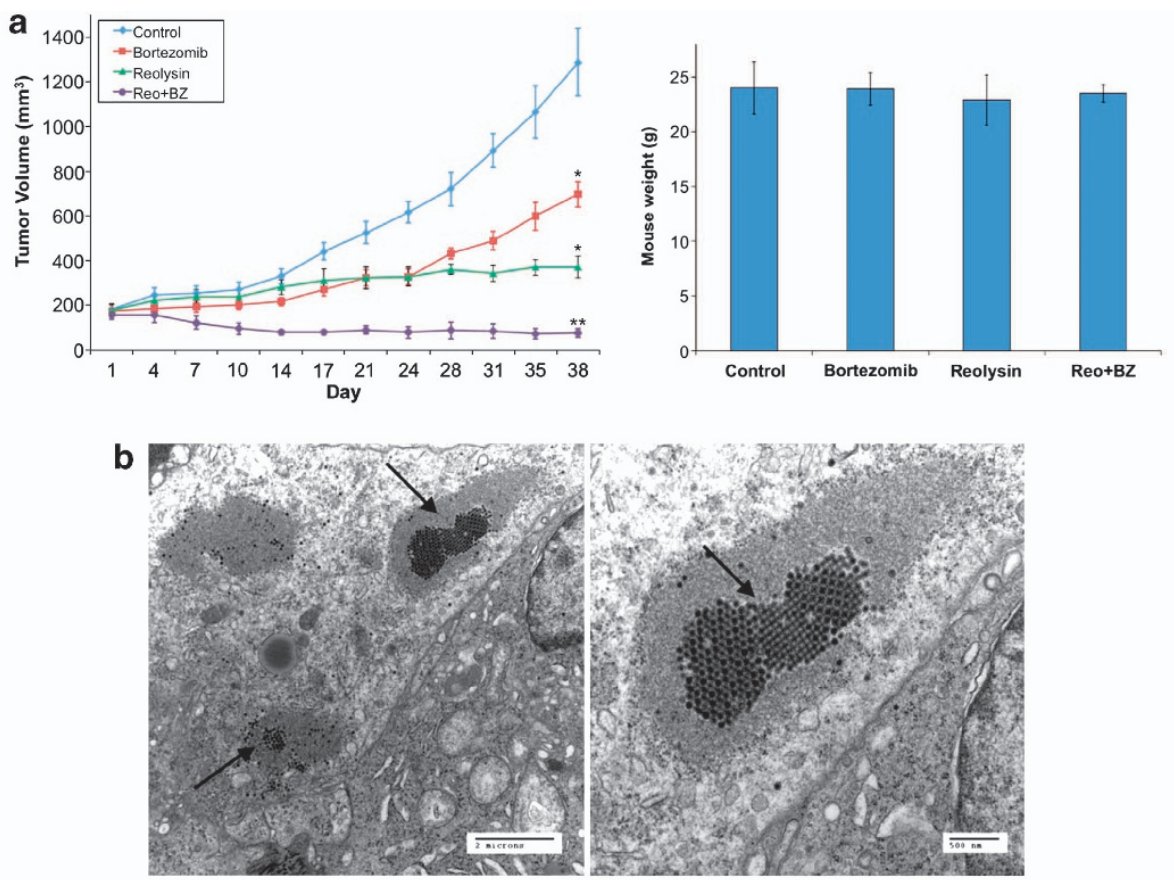

C
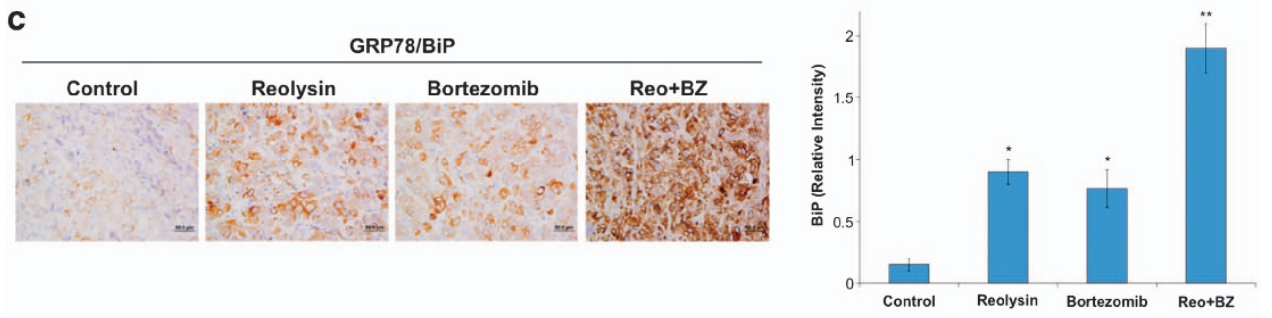

d

TUNEL
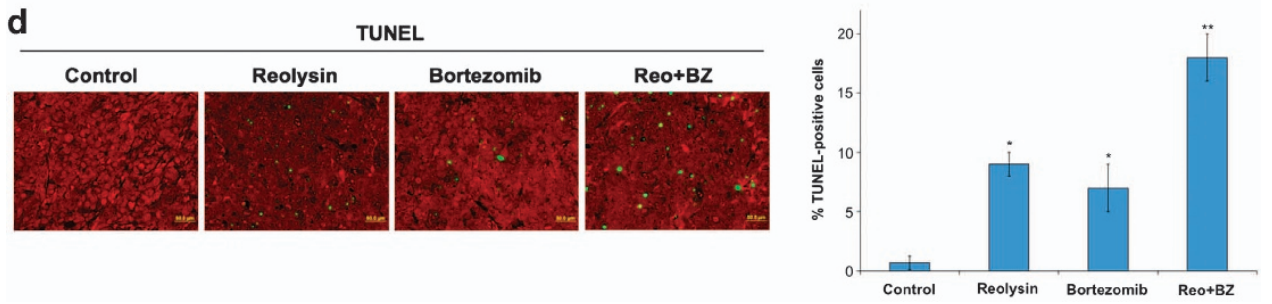

Figure 6 The combination of Reolysin and BZ strongly reduces tumor burden in the Panc- 1 xenograft model. (a) Panc- 1 cells $\left(1 \times 10^{7}\right.$ per mouse) were injected into the flanks of nude mice. When tumors reached approximately $150 \mathrm{~mm}^{3}$ in size, mice were randomized into groups and treated with $0.5 \mathrm{mg} \mathrm{BZ} \mathrm{per} \mathrm{kg} \mathrm{Q3D}, 5 \times 10^{8} \mathrm{TCID}_{50}$ Reolysin Q7D, or both agents for 5 weeks. Tumors were measured twice weekly. Mean \pm S.E.M., $n=8$. *Indicates a significant difference compared with vehicle, or **indicates a significant difference compared with either single-agent treatment. $P<0.05$. Reolysin and BZ are well tolerated in vivo. Animal body weight was determined at the end of the study (day 38) to quantify drug-induced weight loss. Mean \pm S.D., $n=8$. (b) Reovirus replicates in tumors in vivo. Electron microscopy was performed on tumors collected from Reolysin-treated animals and revealed the presence of reovirus. Images shown were taken from an animal treated with the Reolysin $+B Z$ combination. Arrows denote the presence of reovirus. Similar results were observed in mice treated with Reolysin alone. (c) Reolysin and BZ increase BiP expression. BiP expression was measured by IHC, and staining intensity was quantified using ImageJ software. *Indicates a significant difference compared with controls, and **denotes a significant difference compared with either single-agent treatment group $(P<0.05)$. (d) Apoptosis was measured by TUNEL staining. Quantification was conducted by manually counting TUNEL-positive cells. Mean \pm S.D., $n=5$. ${ }^{*}$ Indicates a significant difference compared with controls, and ** represents a significant difference compared with single-agent treatments $P<0.05$

of the oncolytic virus, rhabdovirus. ${ }^{32,33}$ In this study, a synthetic lethal RNAi screen identified that rhabdovirus induced a cytoprotective ER stress response through signaling via the ATF6 and IRE1 pathways. Mild induction of ER stress activates the UPR that results in increased expression of chaperone proteins to interact with misfolded proteins, inhibition of protein synthesis, and enhanced proteasome degradation, which collectively relieve ER stress. ${ }^{34,35}$ However, high levels of ER stress result in apoptosis through activation of caspase-4 or caspase-12. Our approach differs from this study in that rather than inhibiting cytoprotective UPR pathways to increase ER stress, we sought to induce high levels of proteotoxicity to overwhelm the UPR, resulting in enhanced ER stress and Reolysin efficacy. Collectively, these studies demonstrate that oncolytic viral cancer therapy can be augmented by other ER stress stimuli.

The antineoplastic activity of reovirus has been investigated in combination with various standards of care agents, 
including cyclophosphamide, gemcitabine, cisplatin, paclitaxel, and radiation therapy in earlier studies. ${ }^{30,36-38}$ A Reolysin-based combination with platinum- and taxanebased therapy has proven to be especially effective and has advanced into a phase III clinical trial in patients with head and neck cancer. ${ }^{11,39-41}$ Although these standard agents have multifaceted mechanisms of action that contribute to their anticancer activity, both have been shown to stimulate ER stress. ${ }^{16,42,43}$ Our data in pancreatic cancer models suggest that platinum- and taxane-triggered ER stress may significantly contribute to the efficacy of this combination. This possibly warrants further investigation.

Although Reolysin has demonstrated promising activity in several clinical trials, reovirus monotherapy may be insufficient to optimally manage aggressive pancreatic tumors. Considering this, Reolysin may ultimately be most effective when used in combination with other forms of cancer therapy. Our results highlight that induction of ER stress is an important component of Reolysin-mediated apoptosis and that further stimuli of this stress response significantly augment Reolysin's activity. Our collective findings indicate that this type of ER-targeted approach may be especially effective against Ras-activated cancers due to the propensity of reovirus to preferentially replicate in these tumors combined with its intrinsic sensitivity to ER stress. Clinical trials on combination therapy of Reolysin with BZ or other agents that induce ER stress are warranted for the treatment of pancreatic cancer and other malignancies that may be hypersensitive to ER stress-mediated apoptosis.

\section{Materials and Methods \\ Animals and cell lines. The human telomerase (hTERT)-immortalized HPNE cell lines modified to express E6/E7 alone and with KRas were obtained from Michel Ouellette (University of Nebraska, Omaha, NE, USA) and cultured as previously described. ${ }^{44}$ Briefly, HPNE cells were cultured in one volume of medium M3, three volumes of glucose-free DMEM, $5 \%$ fetal bovine serum (FBS), $5.5 \mathrm{mM}$ glucose, $10 \mathrm{ng} / \mathrm{ml} \mathrm{EGF}$, and $50 \mu \mathrm{g} / \mathrm{ml}$ gentamycin. Medium M3 is a defined proprietary formulation optimized for the growth of neuroendocrine cells (InCell Corp., San Antonio, TX, USA). Panc-1, ASPC-1, Capan-2, and CFPAC-1 pancreatic cancer cell lines and L929 murine fibrosarcoma cells were obtained from the American Type Culture Collection (Rockville, MD, USA) and maintained in RPMI supplemented with $10 \%$ FBS. Female nude mice (BALB/c background) were purchased from Harlan (Indianapolis, IN, USA).}

Antibodies and chemicals. Antibodies were obtained from the following commercial sources: anti-tubulin (Sigma, St. Louis, MO, USA); anti-cleaved caspase-3, anti-caspase-12, anti-PERK, anti-p-PERK, anti-p-eif2 $\alpha$, and anti-eif2 $\alpha$ (Cell Signaling Technology, Beverly, MA); anti-GRP78/BiP, antiPDI, anti-ERp57, and anti-calreticulin (Epitomics, Burlingame, CA, USA) anti-caspase-4 (Assay Designs, Ann Arbor, MI, USA); and anti-ubiquitin, anti-GADD34, and anti-KRas (Santa Cruz Biotechnology, Santa Cruz, CA, USA). Anti-reovirus antibody and Reolysin were kindly provided by Oncolytics Biotech Inc. (Calgary, AB, Canada). Horseradish peroxidase-conjugated secondary antibodies for immunoblotting were obtained from Amersham Pharmacia Biotech (Piscataway, NJ, USA). Alexa Fluor 488 rabbit anti-goat and Alexa Fluor 594 goat anti-mouse were obtained from Molecular Probes (Eugene, OR, USA). BZ was purchased from the Cancer Therapy and Research Center (CTRC) pharmacy (San Antonio, TX, USA). Tunicamycin and brefeldin A were obtained from Sigma.

Immunocytochemistry. Pancreatic cells were plated on chamber slides prior to Reolysin or BZ exposure. Cells were fixed with $4 \%$ paraformaldehyde, permeabilized using $0.2 \%$ Triton X-100, and incubated overnight with anti-reovirus or anti-ubiquitin antibodies. Fluorescent secondary antibodies were used to visualize protein localization. Images were captured using an Olympus fluorescent microscope (Olympus, Center Valley, PA, USA) with a DP71 camera and a $\times 40$ objective.

Transmission electron microscopy. Cells were treated with Reolysin or $\mathrm{BZ}$ for $48 \mathrm{~h}$ and processed for electron microscopy. Sections were cut in an LKB Ultracut microtome (Leica, Deerfield, IL, USA), stained with uranyl acetate and lead citrate, and examined in a JEM 1230 transmission electron microscope (JEOL USA Inc., Peabody, MA, USA)

Immunoblotting. Cell pellets were harvested and lysed using Triton X-100 lysis buffer (1\% Triton X-100, $150 \mathrm{mM} \mathrm{NaCl}, 25 \mathrm{mM}$ Tris (pH 7.5)). Approximately $50 \mu \mathrm{g}$ of total cellular protein from each sample were subjected to sodium dodecyl sulfate polyacrylamide gel electrophoresis. Proteins were transferred to nitrocellulose membranes, and the membranes were blocked with $5 \%$ nonfat milk in a Tris-buffered saline solution containing $0.1 \%$ Tween-20 for $1 \mathrm{~h}$. The blots were then probed overnight with relevant antibodies, washed, and probed with species-specific secondary antibodies coupled to horseradish peroxidase. Immunoreactive material was detected by enhanced chemiluminescence (Protein Simple, Santa Clara, CA, USA). Densitometry analysis to quantify band intensity was performed using an Alpha Innotech FluorChem HD2 gel documentation system (Alpha Innotech, Santa Clara, CA, USA).

Quantification of drug-induced cytotoxicity. Cell viability was assessed by MTT assay and quantified using a BioTek microplate reader (BioTek, Winooski, VT, USA). Pro-apoptotic effects were quantified by PI staining and FACS analysis of sub- $G_{0} / G_{1}$ DNA content as previously described. ${ }^{45}$

siRNA experiments. Caspase-4 and non-target SMARTpool siRNA were obtained from Dharmacon (Lafayette, CO, USA). Cells were transfected with $100 \mathrm{nM}$ of each siRNA using Oligofectamine (Invitrogen, Carlsbad, CA, USA) according to the manufacturer's protocol. Transfected cells were incubated for $24 \mathrm{~h}$ and then treated with Reolysin, BZ, or the combination for $48 \mathrm{~h}$. Efficiency of caspase- 4 knockdown was measured at $48 \mathrm{~h}$ by immunoblotting.

Quantitative real-time polymerase chain reaction. CDNA from Reolysin- or BZ-treated cells was used for relative quantification by RT-PCR analyses. cDNA synthesis was performed from $1 \mu \mathrm{g}$ RNA in a 20- $\mu$ reaction mixture using the high-capacity CDNA Reverse Transcription Kit (Applied Biosystems, Foster City, CA, USA). CHOP (DDIT3), GADD34 (PPP1R15A), GRP78/BiP (HSPA5), calreticulin (CALR), PDI (P4HB), ERp57 (PDIA3), and GAPDH or $\beta$-actin transcripts were amplified using commercially available TaqMan Gene expression assays (Applied Biosystems). XBP-1s qRT-PCR was designed to span the 26-bp intron to anneal only the spliced mRNA. Primers and TaqMan probe sequences are as follows: forward primer: $5^{\prime}$-cctggttgctgaagaggag$3^{\prime}$; reverse primer: $5^{\prime}$-agtcaataccgccagaatcc-3'; probe: $5^{\prime}($ Fam)-cctgcacctgctgcggactc- $3^{\prime}$ (Tamra). Relative gene expression was calculated with the $2^{-} \Delta \Delta^{C}{ }_{t}$ method using GAPDH as a housekeeping gene.

Measurement of intracellular $\mathbf{C a}^{2+}$ levels. Panc- 1 and CFPAC-1 cells were treated with Reolysin, BZ, or both for $16 \mathrm{~h}$. Cells were collected, washed in PBS, and incubated with $1 \mu \mathrm{mol} / /$ calcium green-1 (Invitrogen) for $30 \mathrm{~min}$. Fluorescence was quantified using a FACSCanto II with CellQuest Pro Software (BD Biosciences, San Jose, CA, USA).

Implantation of tumor cells and treatment schedule. Panc-1 pancreatic cancer cells were harvested from culture flasks and transferred to serum-free HBSS. Tumor cells $\left(1 \times 10^{7}\right.$ cells) were injected into the right flank of female nude mice and allowed to establish tumors. Following tumor formation, animals were pair matched by tumor size and placed into groups of eight mice. Animals were then treated by i.v. injection of $0.5 \mathrm{mg} \mathrm{BZ}$ per kg every $72 \mathrm{~h}, 5 \times 10^{8}$ $\mathrm{TCID}_{50}$ Reolysin once a week, or both agents for 5 weeks. Tumor volume and animal weight measurements were recorded twice weekly. Tumor tissue was collected for immunohistochemistry $(\mathrm{IHC})$ and electron microscopy at the end of the study.

Terminal deoxyribonucleotide transferase-mediated dUTP nick end labeling assay. DNA fragmentation in tumor samples was analyzed using an FITC-labeled terminal deoxyribonucleotide transferase-mediated dUTP 
nick end labeling (TUNEL) assay kit (Promega, Madison, WI, USA) according to the manufacturer's instructions. PI was used to counterstain the nucleus. Images were captured with an Olympus fluorescent microscope (Olympus) with a DP71 camera and a $\times 20$ objective. Percentages of TUNEL-positive cells were determined by manual counting of five random fields per section.

Immunohistochemistry. Paraffin-embedded tumor sections were deparaffinized in xylene and a graded series of alcohol and rehydrated in PBS. Heat-induced epitope retrieval on paraffin-embedded sections was performed by microwaving slides in a citrate buffer for $5 \mathrm{~min}$. Endogenous peroxides were blocked with a $3 \%$ hydrogen peroxide solution for $10 \mathrm{~min}$. Slides were placed in a protein block solution (5\% horse and $1 \%$ goat serum in PBS) for $20 \mathrm{~min}$, followed by incubation with GRP78/BiP antibody at $4{ }^{\circ} \mathrm{C}$ overnight. After washing with PBS, slides were incubated in a goat anti-rabbit HRP-conjugated secondary antibody for $1 \mathrm{~h}$ at ambient temperature. Positive reactions were visualized using $3,3^{\prime}$-diaminobenzidine (Dako, Carpinteria, CA, USA) for $10 \mathrm{~min}$. The slides were rinsed with water and counterstained with Gill's hematoxylin (Sigma). Images were captured using an Olympus fluorescent microscope with a DP71 camera and a $\times 20$ objective. Image-Pro Plus software version 6.2.1 (MediaCybernetics, Bethesda, MD, USA) was used for image acquisition. ImageJ software was used for quantification of GRP78/BiP levels by densitometric analysis of five random fields containing viable tumor cells.

Statistical analyses. Statistical significance of differences observed between samples was determined using the Tukey-Kramer comparison test or the Student's $t$-test. Differences were considered significant in all experiments at $P<0.05$.

\section{Conflict of Interest}

The authors declare no conflict of interest.

Acknowledgements. This study was supported by the Voelcker Fund Young Investigator Award, the William and Ella Owens Foundation, and the National Cancer Institute P30 Cancer Center Support (Grant No. CA054174). MC is an employee of Oncolytics Biotech Inc.

1. Bos JL. Ras oncogenes in human cancer: a review. Cancer Res 1989; 49: 4682-4689.

2. Fearon ER. K-ras gene mutation as a pathogenetic and diagnostic marker in human cancer. J Natl Cancer Inst 1993; 85: 1978-1980.

3. Malumbres M, Barbacid M. RAS oncogenes: the first 30 years. Nat Rev Cancer 2003; 3: 459-465.

4. Kelly K, Nawrocki S, Mita A, Coffey M, Giles FJ, Mita M. Reovirus-based therapy for cancer. Expert Opin Biol Ther 2009; 9: 817-830.

5. Maitra R, Ghalib MH, Goel S. Reovirus: a targeted therapeutic-progress and potential. $\mathrm{Mol}$ Cancer Res 2012; 10: 1514-1525.

6. Norman KL, Lee PW. Reovirus as a novel oncolytic agent. J Clin Invest 2000; 105: 1035-1038.

7. Comins C, Spicer J, Protheroe A, Roulstone V, Twigger K, White CM et al. REO-10: a phase I study of intravenous reovirus and docetaxel in patients with advanced cancer. Clin Cancer Res 2010; 16: 5564-5572.

8. Forsyth P, Roldan G, George D, Wallace C, Palmer CA, Morris D et al. A phase I trial of intratumoral administration of reovirus in patients with histologically confirmed recurrent malignant gliomas. Mol Ther 2008; 16: 627-632.

9. Harrington KJ, Vile RG, Melcher A, Chester J, Pandha HS. Clinical trials with oncolytic reovirus: moving beyond phase I into combinations with standard therapeutics. Cytokine Growth Factor Rev 2010; 21: 91-98.

10. Morris DG, Feng X, Difrancesco LM, Fonseca K, Forsyth PA, Paterson AH et al. REO-001: a phase I trial of percutaneous intralesional administration of reovirus type 3 dearing (Reolysin(R)) in patients with advanced solid tumors. Invest New Drugs 2012; 31: 696-706.

11. Vidal L, Pandha HS, Yap TA, White CL, Twigger K, Vile RG et al. A phase I study of intravenous oncolytic reovirus type 3 Dearing in patients with advanced cancer. Clin Cancer Res 2008; 14: 7127-7137.

12. Coffey MC, Strong JE, Forsyth PA, Lee PW. Reovirus therapy of tumors with activated Ras pathway. Science 1998; 282: 1332-1334.

13. Strong JE, Coffey MC, Tang D, Sabinin P, Lee PW. The molecular basis of viral oncolysis: usurpation of the Ras signaling pathway by reovirus. Embo J 1998; 17: 3351-3362.

14. Nawrocki ST, Carew JS, Dunner K Jr., Boise LH, Chiao PJ, Huang P et al. Bortezomib inhibits PKR-like endoplasmic reticulum (ER) kinase and induces apoptosis via ER stress in human pancreatic cancer cells. Cancer Res 2005; 65: 11510-11519.
15. Nawrocki ST, Carew JS, Pino MS, Highshaw RA, Andtbacka RH, Dunner K Jr. et al. Aggresome disruption: a novel strategy to enhance bortezomib-induced apoptosis in pancreatic cancer cells. Cancer Res 2006; 66: 3773-3781.

16. Nawrocki ST, Carew JS, Pino MS, Highshaw RA, Dunner K Jr., Huang P et al. Bortezomib sensitizes pancreatic cancer cells to endoplasmic reticulum stress-mediated apoptosis. Cancer Res 2005; 65: 11658-11666.

17. Norman KL, Hirasawa K, Yang AD, Shields MA, Lee PW. Reovirus oncolysis: the Ras/ RalGEF/p38 pathway dictates host cell permissiveness to reovirus infection. Proc Natl Acad Sci USA 2004; 101: 11099-11104.

18. Shmulevitz M, Marcato P, Lee PW. Unshackling the links between reovirus oncolysis, Ras signaling, translational control and cancer. Oncogene 2005; 24: 7720-7728.

19. Hitomi J, Katayama T, Eguchi $Y$, Kudo T, Taniguchi M, Koyama $Y$ et al. Involvement of caspase-4 in endoplasmic reticulum stress-induced apoptosis and Abeta-induced cell death. J Cell Biol 2004; 165: 347-356.

20. Meister S, Schubert U, Neubert K, Herrmann K, Burger R, Gramatzki M et al. Extensive immunoglobulin production sensitizes myeloma cells for proteasome inhibition. Cancer Res 2007; 67: 1783-1792.

21. Obeng EA, Carlson LM, Gutman DM, Harrington WJ Jr., Lee KP, Boise LH. Proteasome inhibitors induce a terminal unfolded protein response in multiple myeloma cells. Blood 2006; 107: 4907-4916.

22. Rao R, Nalluri S, Fiskus W, Savoie A, Buckley KM, Ha K et al. Role of C/EBP homologous protein (CHOP) in panobinostat-mediated potentiation of bortezomibinduced lethal ER stress in mantle cell lymphoma cells. Clin Cancer Res 2010; 16: 4742-4754.

23. Nakagawa T, Zhu H, Morishima N, Li E, Xu J, Yankner BA et al. Caspase-12 mediates endoplasmic-reticulum-specific apoptosis and cytotoxicity by amyloid-beta. Nature 2000; 403: $98-103$

24. Etoh T, Himeno Y, Matsumoto T, Aramaki M, Kawano K, Nishizono A et al. Oncolytic viral therapy for human pancreatic cancer cells by reovirus. Clin Cancer Res 2003; 9: 1218-1223.

25. Hashiro G, Loh PC, Yau JT. The preferential cytotoxicity of reovirus for certain transformed cell lines. Arch Virol 1977; 54: 307-315

26. Pataer A, Hu W, Xiaolin L, Chada S, Roth JA, Hunt KK et al. Adenoviral endoplasmic reticulum-targeted mda-7/interleukin-24 vector enhances human cancer cell killing. $\mathrm{Mol}$ Cancer Ther 2008; 7: 2528-2535.

27. Denoyelle C, Abou-Rjaily G, Bezrookove V, Verhaegen M, Johnson TM, Fullen DR et al. Anti-oncogenic role of the endoplasmic reticulum differentially activated by mutations in the MAPK pathway. Nat Cell Biol 2006; 8: 1053-1063.

28. Hu Y, Lu W, Chen G, Wang P, Chen Z, Zhou Y et al. K-ras(G12V) transformation leads to mitochondrial dysfunction and a metabolic switch from oxidative phosphorylation to glycolysis. Cell Res 2012; 22: 399-412.

29. Lolkema MP, Arkenau HT, Harrington K, Roxburgh P, Morrison R, Roulstone V et al. A phase I study of the combination of intravenous reovirus type 3 Dearing and gemcitabine in patients with advanced cancer. Clin Cancer Res 2011; 17: 581-588.

30. Pandha HS, Heinemann L, Simpson GR, Melcher A, Prestwich R, Errington F et al. Synergistic effects of oncolytic reovirus and cisplatin chemotherapy in murine malignant melanoma. Clin Cancer Res 2009; 15: 6158-6166.

31. Twigger K, Vidal L, White CL, De Bono JS, Bhide S, Coffey M et al. Enhanced in vitro and in vivo cytotoxicity of combined reovirus and radiotherapy. Clin Cancer Res 2008; 14: 912-923.

32. Mahoney DJ, Lefebvre C, Allan K, Brun J, Sanaei CA, Baird $S$ et al. Virus-tumor interactome screen reveals ER stress response can reprogram resistant cancers for oncolytic virus-triggered caspase-2 cell death. Cancer Cell 2011; 20: 443-456.

33. McConkey DJ. RAIDDing ER stress for oncolytic viral therapy. Cancer Cell 2011; 20: 416-418.

34. Gorman AM, Healy SJ, Jager R, Samali A. Stress management at the ER: regulators of ER stress-induced apoptosis. Pharmacol Ther 2012; 134: 306-316.

35. Healy SJ, Gorman AM, Mousavi-Shafaei P, Gupta S, Samali A. Targeting the endoplasmic reticulum-stress response as an anticancer strategy. Eur J Pharmacol 2009; 625: 234-246.

36. Qiao J, Wang H, Kottke T, White C, Twigger K, Diaz RM et al. Cyclophosphamide facilitates antitumor efficacy against subcutaneous tumors following intravenous delivery of reovirus. Clin Cancer Res 2008; 14: 259-269.

37. Sei S, Mussio JK, Yang QE, Nagashima K, Parchment RE, Coffey MC et al. Synergistic antitumor activity of oncolytic reovirus and chemotherapeutic agents in non-small cell lung cancer cells. Mol Cancer 2009; 8: 47.

38. Smakman N, van der Bilt JD, van den Wollenberg DJ, Hoeben RC, Borel Rinkes $\Perp$ Kranenburg O. Immunosuppression promotes reovirus therapy of colorectal liver metastases. Cancer Gene Ther 2006; 13: 815-818.

39. Karapanagiotou EM, Roulstone V, Twigger K, Ball M, Tanay M, Nutting C et al. Phase I/II trial of carboplatin and paclitaxel chemotherapy in combination with intravenous oncolytic reovirus in patients with advanced malignancies. Clin Cancer Res 2012; 18 : 2080-2089.

40. Roulstone V, Twigger K, Zaidi S, Pencavel T, Kyula JN, White C et al. Synergistic cytotoxicity of oncolytic reovirus in combination with cisplatin-paclitaxel doublet chemotherapy. Gene Ther 2012; 20: 521-528. 
41. Twigger K, Roulstone V, Kyula J, Karapanagiotou EM, Syrigos KN, Morgan R et al. Reovirus exerts potent oncolytic effects in head and neck cancer cell lines that are independent of signalling in the EGFR pathway. BMC Cancer 2012; 12: 368.

42. Liao PC, Tan SK, Lieu CH, Jung HK. Involvement of endoplasmic reticulum in paclitaxelinduced apoptosis. J Cell Biochem 2008; 104: 1509-1523.

43. Mandic A, Hansson J, Linder S, Shoshan MC. Cisplatin induces endoplasmic reticulum stress and nucleus-independent apoptotic signaling. J Biol Chem 2003; 278: 9100-9106.

44. Zhao S, Wang Y, Cao L, Ouellette MM, Freeman JW. Expression of oncogenic K-ras and loss of Smad4 cooperate to induce the expression of EGFR and to promote invasion of immortalized human pancreas ductal cells. Int J Cancer 2010; 127: 2076-2087.
45. Kelly KR, Espitia CM, Mahalingam D, Oyajobi BO, Coffey M, Giles FJ et al. Reovirus therapy stimulates endoplasmic reticular stress, NOXA induction, and augments bortezomib-mediated apoptosis in multiple myeloma. Oncogene 2012; 31: 3023-3038.

(c) (i) $(-)($ Cell Death and Disease is an open-access journal published by Nature Publishing Group. This work is licensed under a Creative Commons Attribution-NonCommercialNoDerivs 3.0 Unported License. To view a copy of this license, visit http://creativecommons.org/licenses/by-nc-nd/3.0/

Supplementary Information accompanies this paper on Cell Death and Disease website (http://www.nature.com/cddis) 